\title{
Evaluation of physical activity and its relationship to health-related quality of life in patients on chronic hemodialysis
}

\author{
Akihiko Katayama $\cdot$ Nobuyuki Miyatake $\cdot$ \\ Hiroyuki Nishi $\cdot$ Kazuhiro Uzike • Noriko Sakano • \\ Hiroo Hashimoto $\cdot$ Kiichi Koumoto
}

Received: 18 October 2013/Accepted: 17 January 2014/Published online: 5 February 2014

(C) The Japanese Society for Hygiene 2014

\begin{abstract}
Objective The aim of this study was to investigate the relationship between physical activity and health-related quality of life (HRQOL) in patients on chronic hemodialysis.

Methods A total of 31 men $(69.0 \pm 11.1$ years $)$ and 17 women (66.9 \pm 10.0 years) among 61 male and 30 female patients on chronic hemodialysis at Innoshima General Hospital, Onomichi, Japan, were enrolled in this crosssectional study. Physical activity was evaluated using triaxial accelerometers. HRQOL and psychological distress were also evaluated using the EuroQol questionnaire (EQ5D) and the K6 questionnaire, respectively.

Results Physical activity evaluated by $\Sigma[$ metabolic equivalents $\times \mathrm{h}$ per week (METs.h/w)] was $8.1 \pm 6.0$ METs.h/w, and EQ-5D score was $0.754 \pm 0.177$. Among all patients, EQ-5D scores were significantly correlated with physical activity over 4 METs on non-hemodialysis treatment days ( $r=0.426, p=0.003$ ). In women, EQ-5D scores were also correlated with physical activity over 4 METs on hemodialysis treatment days and non-hemodialysis treatment days. By stepwise multiple regression analysis, physical activity over 4 METs on non-hemodialysis treatment days was a determinant factor of EQ-5D even after adjusting for age and K6 scores.
\end{abstract}

\footnotetext{
A. Katayama $(\bowtie) \cdot$ N. Miyatake $\cdot$ N. Sakano Department of Hygiene, Faculty of Medicine, Kagawa University, 1750-1, Miki, Kagawa 761-0793, Japan e-mail: kata@med.kagawa-u.ac.jp

H. Nishi $\cdot$ K. Uzike $\cdot$ H. Hashimoto $\cdot$ K. Koumoto Innoshima General Hospital, Onomichi,

Hiroshima 722-2323, Japan
}

Conclusion Physical activity over 4 METs on non-hemodialysis treatment days might be associated with EQ-5D in patients on chronic hemodialysis, especially in women.

Keywords Hemodialysis - Health-related quality of life (HRQOL) · EuroQol (EQ-5D) · Psychological distress · Physical activity

\section{Introduction}

Patients on hemodialysis are dramatically increasing and this has become a public health challenge in Japan. Over 300,000 patients are undergoing hemodialysis [1]. Therefore, adequate management of patients on chronic hemodialysis is urgently required.

Patients on chronic hemodialysis are thought to be much less active in their daily lives than healthy subjects, and lower physical activity may affect the various clinical parameters in patients on chronic hemodialysis. Recently, a very small tri-axial accelerometer called the Actimarker (Panasonic, Osaka, Japan) has been developed [2, 3]. This accelerometer can monitor physical activity continuously for over 1 month, and it can be easily used by simply attaching it to the waist.

In addition, in recent years, health-related quality of life (HRQOL) has become a prevalent concept worldwide. The EuroQol questionnaire (EQ-5D) [4-6], which is a widely used questionnaire evaluating HRQOL, has been translated into Japanese, and an official version was developed in May 1998 [7, 8]. However, the relationship between physical activity accurately evaluated by tri-accelerometer and HRQOL still remains to be investigated in patients on chronic hemodialysis in Japan. Therefore, in this study, we evaluated the effect of physical activity on HRQOL using 
EQ-5D in patients on chronic hemodialysis in a crosssectional study.

\section{Subjects and methods}

\section{Subjects}

We used data on 31 men $(69.0 \pm 11.1$ years $)$ and 17 women (66.9 \pm 10.0 years) among 91 patients who met the following criteria: (1) wanted to be enrolled voluntarily in this cross-sectional study at Innoshima General Hospital, Onomichi, Japan, (2) received anthropometric parameter, physical activity, HRQOL, and psychological distress measurements, and (3) provided written informed consent (Table 1).

Ethical approval for the study was obtained from the Ethical Committee of Innoshima General Hospital, Onomichi, Japan (Innoshima General Hospital H24-3-28).

Anthropometric and body composition measurements

Anthropometric and body compositions were evaluated based on the following parameters: height, body weight, and body fat percentage. Body mass index (BMI) was calculated by weight/(height $)^{2}\left(\mathrm{~kg} / \mathrm{m}^{2}\right)$. The body fat percentage was measured by the impedance method (MLT-50, SEKISUI MEDICAL CO. LTD., Tokyo, Japan).

Physical activity

In this study, we used the Actimarker, a small and lightweight $(36.0 \mathrm{~g})$ accelerometer which can continuously monitor physical activity for over one month. It can be easily used by simply attaching it to the waist. Actimarker collects tri-axial acceleration data at $20 \mathrm{~Hz}$. Furthermore, the standard deviation of the data of $1 \mathrm{~min}$ is defined as a average value of acceleration. The METs value can be calculated using a linear regression formula of the relationship between the average value of acceleration and the METs value measured by the gas metabolic system $[2,3]$. The subjects were taught how to use the instrument, and were told to wear it on their belt or waist band at the right midline of the thigh from the moment they awoke until they went to bed, except while bathing or swimming, for seven consecutive days. The activity monitor was firmly attached to their clothes at the waist by a clip as previously described [9].

\section{EQ-5D score}

EQ-5D is a brief, self-completed instrument for describing and valuing quality of health status defined by the EQ-5D scores. This descriptive system classifies respondents into one of 243 distinct health states. The descriptive system consists of five dimensions: (1) mobility, (2) self-care, (3) usual activities, (4) pain/discomfort, and (5) anxiety/ depression. Each dimension has three levels, allowing for $3^{5}$ (i.e., 243) possible health combinations. In addition, for completeness, the states "dead" and "unconscious" were also incorporated in the framework [4-8]. The unique EQ$5 \mathrm{D}$ health state is defined by combining one level from each of the five dimensions and producing a set of utility values for 245 health states. We used the Japanese EQ-5D instrument to assess the QOL of the subjects [8, 10].

\section{The K6 score}

As shown in previous reports [11], the K6 was used as an index of psychological distress [12, 13]. The questionnaire consisted of six questions. "Over the last month, how often did you feel: (1) nervous, (2) hopeless, (3) restless or fidgety, (4) so sad that nothing could cheer you up, (5) that everything was an effort, (6) worthless?" The subjects were requested to respond by choosing from the following: "all of the time" (4 points), "most of the time" (3 points), "some of the time" ( 2 points), "a little of the time" (1 point), and "none of the time" ( 0 points). The total point score range was $0-24$. The $\mathrm{K} 6$ is based on modern psychometric measurement theory. In the field of psychometric current, the K6 is an excellent measurement method [14-16]. Using the standard back-translation method, the Japanese version of K6 has been developed, and it has been validated [12]. As suggested by Kessler et al. [16], we have classified subjects with scores of 13 points or more.

\section{Statistical analysis}

Data are expressed as mean \pm standard deviation (SD) values. Simple correlation analysis was performed as well to test for the significance of the linear relationship among continuous variables, where $p<0.05$ was considered to be statistically significant. In addition, stepwise multiple regression analysis was also performed on factors affecting HRQOL.

\section{Results}

Written informed consent was obtained from 72 patients, and clinical profiles of enrolled patients on chronic hemodialysis for analysis (31 men and 17 women) are summarized in Table 1. Physical activity evaluated by $\Sigma$ [metabolic equivalents $\times \mathrm{h}$ per week $(\mathrm{MET} \cdot \mathrm{h} / \mathrm{w})]$ was $8.1 \pm 6.0 \mathrm{METs} \cdot \mathrm{h} / \mathrm{w}$ (0.5-29.9). EQ-5D scores and K6 scores were $0.754 \pm 0.177$ and $3.6 \pm 3.7$, respectively, among all patients on chronic hemodialysis. 


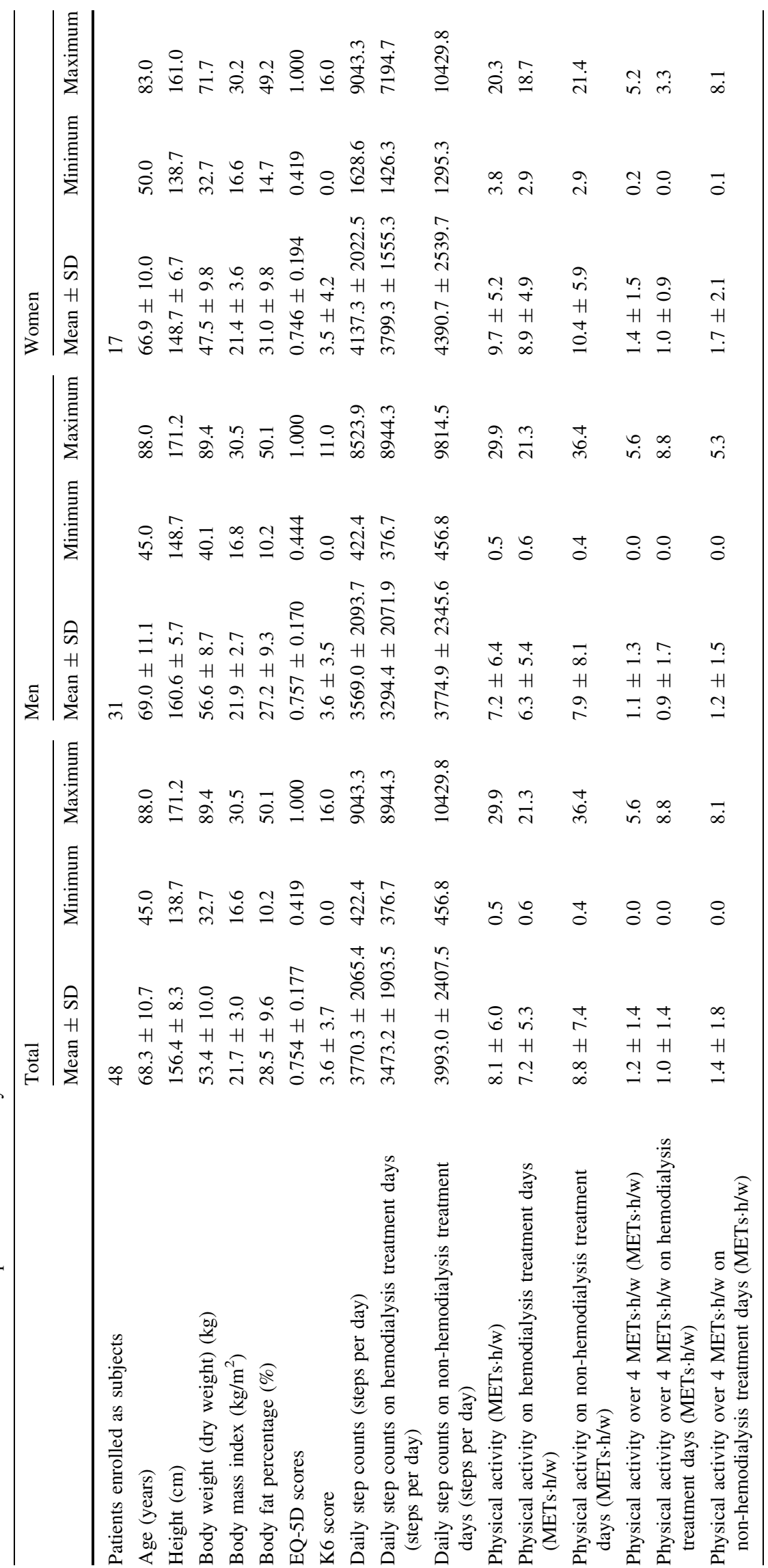


Table 2 Simple correlation analysis between EQ-5D scores and physical activity in patients on hemodialysis

\begin{tabular}{|c|c|c|c|c|c|c|}
\hline & \multicolumn{2}{|l|}{ Total } & \multicolumn{2}{|l|}{ Men } & \multicolumn{2}{|c|}{ Women } \\
\hline & $r$ & $p$ & $r$ & $p$ & $r$ & $p$ \\
\hline Daily step counts (steps per day) & 0.204 & 0.164 & 0.317 & 0.830 & 0.032 & 0.904 \\
\hline Daily step counts on hemodialysis treatment days (steps per day) & 0.146 & 0.321 & 0.182 & 0.328 & 0.095 & 0.718 \\
\hline Daily step counts on non-hemodialysis treatment days (steps per day) & 0.220 & 0.133 & 0.374 & $\mathbf{0 . 0 3 8}$ & 0.001 & 0.998 \\
\hline Physical activity (METs $\cdot h / w)$ & 0.268 & 0.066 & 0.219 & 0.237 & 0.413 & 0.099 \\
\hline Physical activity on hemodialysis treatment days (METs $\cdot \mathrm{h} / \mathrm{w})$ & 0.159 & 0.164 & 0.740 & 0.693 & 0.350 & 0.169 \\
\hline Physical activity on non-hemodialysis treatment days (METs $\cdot h / w)$ & 0.297 & 0.040 & 0.266 & 0.148 & 0.417 & 0.096 \\
\hline Physical activity over 4 METs.h/w (METs $\cdot h / w)$ & 0.355 & 0.013 & 0.154 & 0.409 & 0.671 & $\mathbf{0 . 0 0 3}$ \\
\hline Physical activity over 4 METs $\cdot \mathrm{h} / \mathrm{w}$ on hemodialysis treatment days $(\mathrm{MET} \cdot \mathrm{h} / \mathrm{w})$ & 0.109 & 0.462 & -0.054 & 0.774 & 0.609 & 0.009 \\
\hline Physical activity over 4 METs $\cdot \mathrm{h} / \mathrm{w}$ on non-hemodialysis treatment days (METs.h/w) & 0.426 & 0.003 & 0.281 & 0.126 & 0.624 & 0.007 \\
\hline
\end{tabular}

Bold values are statistically significant $(p<0.05)$

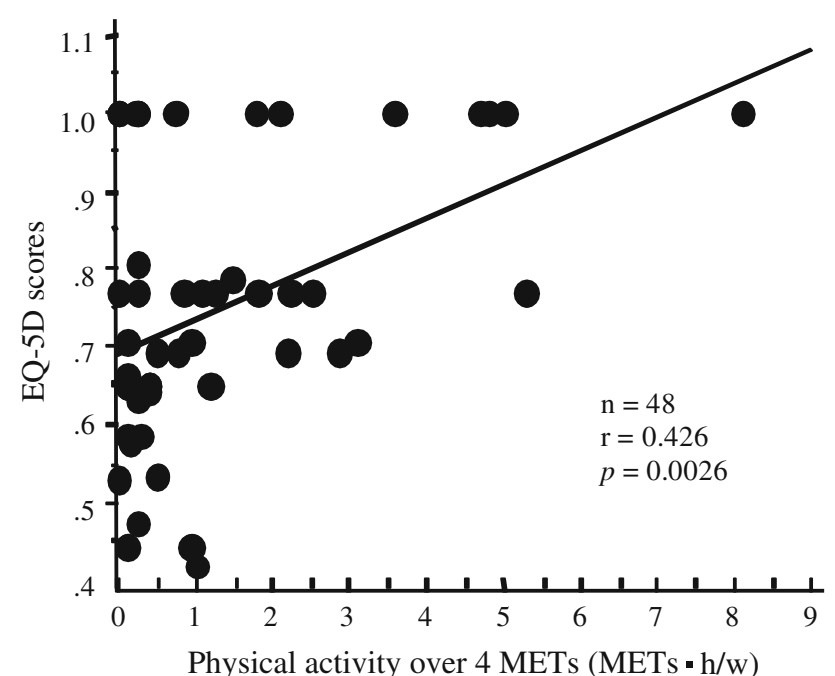

Fig. 1 Simple correlation analysis between EQ-5D scores and physical activity over 4 METs on non-hemodialysis treatment days $(\mathrm{METs} \cdot \mathrm{h} / \mathrm{w})$ in all patients

We evaluated the relationship between EQ-5D scores and physical activity measured by the Actimarker (Table 2). EQ-5D scores were positively and significantly correlated with physical activity over 4 METs on non-hemodialysis treatment days $(r=0.426, p=0.003)$ among all patients (Fig. 1). In women, EQ-5D scores were positively and significantly correlated with physical activity over 4 METs on hemodialysis treatment days and non-hemodialysis treatment days (Table 2). However, in men, a clear relationship between EQ-5D and physical activity was not noted.

Psychological distress might affect HRQOL. Therefore, we also investigated the relationship between the K6 scores and physical activity (Table 3 ). The K6 scores were negatively and significantly correlated with physical activity on hemodialysis treatment days in women $(r=-0.491$, $p=0.045)$.

Finally, we performed stepwise regression analysis, and used EQ-5D scores as the dependent variable, and age, K6 scores, physical activity on hemodialysis treatment days and non-hemodialysis treatment days, and physical activity over 4 METs on hemodialysis treatment days and non-hemodialysis treatment days, as independent variables to adjust for confounding factors. Among all patients on chronic hemodialysis, only physical activity over 4 METs on non-hemodialysis treatment days was a determinant factor of EQ-5D [EQ-5D scores $=0.043$ (physical activity over 4 METs on non-hemodialysis treatment days $\left.)+0.695, r^{2}=0.181, p=0.0026\right]$.

\section{Discussion}

We first evaluated the relationship between HRQOL and physical activity using a tri-axial accelerometer in Japanese patients on chronic hemodialysis in a cross-sectional study. A level of 4 METs and over might be associated with EQ$5 \mathrm{D}$ scores, especially in women.

Wong et al. [17] reported that, using the global physical activity questionnaire (GPAQ), none of the hemodialysis patients had high physical activity levels. Johansen et al. [18] also reported that patients on hemodialysis were less active than healthy sedentary controls, and this difference was more pronounced among older subjects using a three-dimensional accelerometer and with an activity questionnaire. In addition, engaging in habitual physical activity among outpatients undergoing maintenance hemodialysis was associated with decreased mortality risk in Japanese patients on chronic hemodialysis for 7 years [19]. In this study, we evaluated the physical activity in patients on chronic hemodialysis using a newly developed tri-axial accelerometer. The average daily step count was $3770.3 \pm 2065.4$ (422.4-9043.3) and that of physical activity was $8.1 \pm 6.0 \mathrm{METs} \cdot \mathrm{h} / \mathrm{w}$ (0.5-29.9). Therefore, the subjects in this study were less active than subjects in previous studies.

There have been some reports on HRQOL in patients on chronic dialysis. In peritoneal dialysis patients, the EQ-5D 
Table 3 Simple correlation analysis between K6 scores and physical activity in patents on hemodialysis

\begin{tabular}{|c|c|c|c|c|c|c|}
\hline & \multicolumn{2}{|l|}{ Total } & \multicolumn{2}{|l|}{ Men } & \multicolumn{2}{|l|}{ Women } \\
\hline & $r$ & $p$ & $r$ & $p$ & $r$ & $p$ \\
\hline Daily step counts (steps per day) & -0.260 & 0.075 & -0.353 & 0.051 & -0.111 & 0.670 \\
\hline Daily step counts on hemodialysis treatment days (steps per day) & -0.227 & 0.122 & -0.323 & 0.076 & -0.040 & 0.880 \\
\hline Daily step counts on non-hemodialysis treatment days (steps per day) & -0.255 & 0.080 & -0.337 & 0.064 & -0.137 & 0.600 \\
\hline Physical activity (METs·h/w) & -0.370 & $\mathbf{0 . 0 1 0}$ & -0.332 & 0.068 & -0.472 & 0.056 \\
\hline Physical activity on hemodialysis treatment days (METs.h/w) & -0.397 & 0.005 & -0.359 & 0.048 & -0.491 & 0.045 \\
\hline Physical activity on non-hemodialysis treatment days (METs.h/w) & -0.314 & $\mathbf{0 . 0 3 0}$ & -0.279 & 0.129 & -0.420 & 0.093 \\
\hline Physical activity over 4 METs.h/w (METs·h/w) & -0.232 & 0.112 & -0.159 & 0.394 & -0.337 & 0.186 \\
\hline Physical activity over 4 METs.h/w on hemodialysis treatment days (METs.h/w) & -0.136 & 0.356 & -0.103 & 0.580 & -0.257 & 0.319 \\
\hline Physical activity over 4 METs $\cdot \mathrm{h} / \mathrm{w}$ on non-hemodialysis treatment days (METs.h/w) & -0.238 & 0.103 & -0.159 & 0.392 & -0.330 & 0.196 \\
\hline
\end{tabular}

Bold values are statistically significant $(p<0.05)$

score was $0.65 \pm 0.23$ in Thai patients [20]. Kusleikaite et al. [21] showed that both baseline HRQOL and decline of HRQOL are independent predictors of mortality in hemodialysis patients using a short form 36 questionnaire (SF36) in a longitudinal analysis. Less severe depression and fewer anxiety symptoms were also associated with higher HRQOL [22], and regular exercise was correlated with more positive patients and fewer depressive symptoms in the Dialysis Outcomes and Practice Patterns Study [23]. In healthy Japanese [24], HRQOL (SF-36 scores) was higher in individuals spending $>25 \%$ of their total activity at an intensity $>3$ METs [24]. We evaluated HRQOL in patients on chronic hemodialysis using EQ-5D and the score was $0.754 \pm 0.177$. It is noteworthy that EQ-5D scores were associated with physical activity over 4 METs (METs.h/w), especially in women. By stepwise regression analysis, the relationship between EQ-5D scores and physical activity over 4 METs on non-hemodialysis treatment days (METs $\mathrm{h} /$ w) still remained even after adjusting confounding factors in all patients. Mustata et al. [25] showed that long-term exercise training improved physical impairment, arterial stiffness, and HRQOL in patients on predialysis. Ota et al. [26] reported that a gymnastics program including stretching and isotonic muscle conditioning using two tennis balls (2-3 METs using a sitting position) improved physical fitness and instrumental activity of daily living (IADL) in elderly hemodialysis patients.

We evaluated the relationship between physical activity and HRQOL in patients on chronic hemodialysis, and physical activity was evaluated on hemodialysis treatment days and non-hemodialysis treatment days. When promoting increased physical activity in patients on chronic hemodialysis, it is generally important to evaluate their situation and fitness level. In addition, physical activity on hemodialysis treatment days and non-hemodialysis treatment days might be meaningful for instructors. However, increasing physical activity may be difficult for patients on chronic hemodialysis, especially on hemodialysis treatment days. In this study, physical activity over 4 METs on nonhemodialysis treatment days was associated with HRQOL in women and total subjects. However, this association was not observed in men. In fact, men were less active and parameters of physical activity in men [over 4 METs on non-hemodialysis treatment days: $1.2 \pm 1.5 \mathrm{METs} \cdot \mathrm{h} / \mathrm{w}$ $(0-5.3)]$ were lower than those in women. Nevertheless, this reference value may be a useful milestone for some patients on chronic hemodialysis.

Potential limitations remain in this study. First, this study was a cross-sectional and not a longitudinal study. Second, 48 patients on chronic hemodialysis in this study voluntarily underwent measurements. Third, we could not identify the mechanism that links physical activity and HRQOL. Fourth, the small sample size, especially of women, makes it difficult to validate this study. We could not analyze the relationship between EQ-5D scores and physical activity by stepwise regression analysis in women. Bayoumi et al. [27] reported that gender differences may affect QOL scores in patients on chronic hemodialysis. In addition, other potential factors that may influence physical activity could not be evaluated accurately in this study.

Nonetheless, it seems reasonable to suggest that promoting physical activity might result in improved HRQOL in some patients on hemodialysis. To demonstrate this clearly, further prospective and detailed studies in patients on chronic hemodialysis are needed.

Conflict of interest There is no conflict of interest.

\section{References}

1. The Japanese Society for Dialysis Therapy. 2013. http://docs.jsdt. or.jp/overview/pdf2013/p003.pdf. Accessed 19 Oct 2013 (in Japanese). 
2. Matsumura Y, Yamamoto M, Kitado T, Nakamura H, Kidera K, Fujimoto S. High-accuracy physical activity monitor utilizing three-axis accelerometer. Nati Tech Rep. 2008;56:60-6 (in Japanese).

3. Hikihara Y, Tanaka S, Ohkawara K, Ishikawa-Takata K, Tabata I. Validation and comparison of 3 accelerometers for measuring physical activity intensity during nonlocomotive activities and locomotive movements. J Phys Act Health. 2012;9:935-43.

4. EuroQol Group. EuroQol; a new facility for the measurement of health-related quality of life. Health Policy. 1990;16:199-208.

5. Rabin R, de Charro F. EQ-5D: a measurement of health status from the EuroQol Group. Ann Med. 2001;33:337-43.

6. Brooks R. EuroQol: the current state of play. Health Policy. 1996;37:53-72.

7. Ikeda S, Ikegami N, on behalf of the Japanese EuroQol Tariff Project. Health status in Japanese population: Results from Japanese EuroQol study. J Health Care Soc. 1999;9:83-92.

8. Ikegami N, Fukuhara S, Ikeda S. QOL evaluation handbook for clinical diagnosis. Tokyo: Igaku Shoin; 2001. p. 45-9 (in Japanese).

9. Sugino A, Minakata Y, Kanda M, Akamatu K, Koarai A, Hirano $\mathrm{T}$, et al. Validation of a compact motion sensor for the measurement of physical activity in patients with chronic obstructive pulmonary disease. Respiration. 2012;83:300-7.

10. Fujikawa A, Suzue T, Jitsunari F, Hirao T. Evaluation of healthrelated of life using EQ-5D in Takamatsu, Japan. Environ Health Prev Med. 2011;16:25-35.

11. Sakano N, Suzue T, Miyakake N, Miyamae Y, Nagatomi T, Yoda $\mathrm{T}$, et al. Factors associated with psychological distress of Public Health Nurse in Kagawa prefecture, Japan: a pilot study. Open J Nurs. 2012;2:23-30.

12. Furukawa TA, Kawakami N, Saitoh M, Ono Y, Nakane Y, Nakamura Y, et al. The performance of the Japanese version of the K6 and K10 in the World Mental Health Survey Japan. Int J Methods Psychiatr Res. 2008;17:152-8.

13. Iwata N, Uno B, Suzuki T. Psychometric properties of the 30 -item version general health questionnaire in Japanese. Jpn J Psychiatry Neurol. 1994;48:547-56.

14. Kessler RC, Andrews G, Colpe LJ, Hiripi E, Mroczek DK, Normand SL, et al. Short screening scales to monitor population prevalences and trends in non-specific psychological distress. Psychol Med. 2002;32:959-96.

15. Furukawa TA, Kessler RC, Slade T, Andrews G. The performance of the K6 and K10 screening scales for psychological distress in the Australian National Survey of Mental Health and Well-Bing. Psychol Med. 2003;33:357-62.
16. Kessler RC, Barker PR, Colpe LJ, Epstein JF, Gfroerer JC, Hiripi $\mathrm{E}$, et al. Screening for serious mental illness in the general population. Aech Gen Psychiatry. 2003;60:184-9.

17. Wong SW, Chan YM, Lim TS. Correlates of physical activity level among hemodialysis patients in Selangor, Malaysia. Malays J Nutr. 2011;17:277-86.

18. Johansen KL, Chertow GM, Ng AV, Mulligan K, Carey S, Schoenfeld PY, et al. Physical activity levels in patients on hemodialysis and healthy sedentary controls. Kidney Int. 2000;57:2564-70.

19. Matsuzawa R, Matsunaga A, Wang G, Kutsuna T, Ishii A, Abe Y, et al. Habitual physical activity measured by accelerometer and survival in maintenance hemodialysis patients. Clin J Am Soc Nephrol. 2012;7:2010-6.

20. Sakthong P, Kasemsup V. Health utility measured with EQ-5D in Thai patients undergoing peritoneal dialysis. Value Health. 2012;15:S79-84.

21. Kusleikaite N, Bumblyte IA, Kuzminskis V, Vaiciuniene R. The associations between health-related quality of life and mortality among hemodialysis patients. Medicina (Kaunas). 2010;46:531-7.

22. McKercher CM, Venn AJ, Blizzard L, Nelson MR, Palmer AJ, Ashby MA, et al. Psychosocial factors in adults with chronic kidney disease: characteristics of pilot participants in the Tasmanian Chronic Kidney Disease study. BMC Nephrol. 2013;14:83.

23. Tentori F, Elder SJ, Thumma J, Pisoni RL, Bommer J, Fissell RB, et al. Physical exercise among participants in the Dialysis Outcomes and Practice Patterns Study (DOPPS): correlates and associated outcomes. Nephrol Dial Transpl. 2010;25:3050-62.

24. Aoyagi Y, Park H, Park S, Shephard RJ. Habitual physical activity and health-related quality of life in older adults: interactions between the amount and intensity of activity (the Nakanojo Study). Qual Life Res. 2010;19:333-8.

25. Mustata S, Groeneveld S, Davidson W, Ford G, Kiland K, Manns B. Effects of exercise training on physical impairment, arterial stiffness and health-related quality of life in patients with chronic kidney disease: a pilot study. Int Urol Nephrol. 2011;43:1133-41.

26. Ota S, Takahashi K, Suzuki H, Nishimura S, Makino H, Ota Z, et al. Exercise rehabilitation for elderly patients on chronic hemodialysis. Geriatr Nephrol Urol. 1996;5:157-65.

27. Bayoumi M, AI Harbi A, AI Suwaida A, AI Ghonaim M, AI Wakeel J, Mishkiry A. Predictors of quality of life in hemodialysis patients. Saudi J Kidney Dis Transpl. 2013;24:254-9. 